\title{
Editorial
}

\section{Call for a Canadian Public Mental Health System: Transformative change amid a global pandemic*}

\author{
Nazilla Khanlou ${ }^{1}$ \\ ${ }^{1}$ York University
}

*This Editorial was previously published in the Journal of Concurrent Disorders, https://concurrentdisorders.ca/ and is reprinted here with permission of the editor.

By now in all corners of the world citizens have heard about the rapidly progressing COVID-19 pandemic. Everyone is affected, directly or indirectly. Those who have passed away have left families devastated while the sick struggle to keep hope amid gasps for air. Our health professionals are our heroes as they provide care for all, including a growing number of patients with Coronavirus related illnesses. Our work is affected, our home lives are affected, our communities are affected, our sports and culture are affected, our news is affected, our global and local economies are affected, our national borders are affected, and yes our politics are affected. Compared to a few months, we are all affected in unimaginable ways.

During this extraordinary time we have relearned about the critical role of our public health system. Public health matters again, as we listen to public health officials remind us of what we must and must not do to stay healthy and to prevent further spread of disease. They provide on a daily basis the grim and growing morbidity and mortality related statistics of the pandemic. Our constant high stress level in this unprecedented era has brought to the foreground the imperative for a public mental health system. Even for those of us with years of mental health research, teaching, and practice experience we recognize that, despite growing public mental health concerns, we are lacking a public mental health system.

Yes good mental health practices and policies exist. Yes there have been impressive gains in public efforts and funding to destigmatize mental illness. More people are talking about the importance of mental health and wellbeing than in the past decades. However, access to and utilization of mental health services is not uniform and is impeded by multiple barriers.

Public mental health promotion and care in Canada, and I would think in most parts of the globe, is not where it can be in 2020. But if we are to take away some lessons learned from the current Coronavirus crisis, when rapid societal changes are taking place, we cannot forget our public's mental health. To do so would result in individual suffering, rules not followed well, community discohesion, and citizenship isolation.

During this challenging time, let's commit to a Public Mental Health System. We desperately need our public health approach to broaden its reach into supporting and promoting our populations' mental wellbeing. Although some publically funded mental health care coverage exists, aimed at the more tertiary end of care, our mental health care system is otherwise fragmented, with patchworks of private funding (if one has full-time employment) and provincial funding covering services (if they exist). Often mental health care services are concentrated in large cities, a challenge to get self-referral to, and sadly timely access is not within reach of all. Multiple barriers impede equitable access. Study after study has identified what these barriers are.

Let's look at this difficult period of unparallel magnitude in recent history as a time also to transform our approach to our mental health and wellbeing. Let's start a public policy dialogue, acknowledging our citizens' real fears and isolation, 
and yet hope and resilience. Let's follow it decisively with action, calling for a Public Mental Health System that is multidisciplinary, person family-centered and inclusive of all of Canada. We need a de-centralized approach to our future Public Mental Health System that works closely with the community sectors in facilitating timely access, long term support, and reintegration to employment, training, and societal involvement. We need all disciplines and sectors and organizations at the planning table of our future Public Mental Health System, not a select few. In every step of the way we need the participation of persons and families with lived experience in its design, implementation, and evaluation.

Despite our current most challenging time, it is possible to transform.

Please Canada, let's not forget mental health.

Bio Nazilla Khanlou, RN, PhD is the Women's Health Research Chair in Mental Health in the Faculty of Health at York University and an Associate Professor in its School of Nursing. She is the Academic Lead of the Lillian Meighen Wright Maternal-Child Health Scholars Program. Professor Khanlou's clinical background is in psychiatric nursing. Her overall program of research is situated in the interdisciplinary field of community-based mental health promotion in general, and mental health promotion among youth and women in multicultural and immigrant-receiving settings in particular. She applies intersectionality-informed frameworks, using diverse research methods, in community based research. She is founder of the International Network on Youth Integration (INYI), an international network for knowledge exchange and collaboration on youth. She has published articles, books, and reports on immigrant youth and women, and mental health.

Original source: Khanlou N. (2020). Editorial: Call for a Canadian Public Mental Health System:

Transformative change amid a global pandemic. Journal of Concurrent Disorders, 2(1), 1-2. Available

url:

https://concurrentdisorders.ca/2020/03/31/call-fora-canadian-public-mental-health-system/ 